in vivo $33: 495-500(2019)$

doi:10.21873/invivo.11501

\title{
Skin Extension by Multiple Incisions (SEMI)
}

\author{
RALPH GAULKE \\ Section Upper Extremity, Foot and Rheuma Surgery, Trauma Department, \\ Medical School Hanover (MHH), Hanover, Germany
}

\begin{abstract}
Background/Aim: There is a need for a simple and atraumatic technique for wound closure in soft tissue defects. The aim of this study was to evaluate the technique of skin extension by multiple incisions in the healing of soft tissue defects following trauma. Patients and Methods: This is a retrospective clinical study on soft tissue defects after trauma. The dimensions of the defects were measured intraoperatively. Exclusion criteria were combined techniques of skin extension by multiple incisions with local flaps or free skin grafts, preoperative numbness, and incomplete data. Complications until wound healing were documented. Results: One hundred soft tissue defects were evaluated. Uneventful healing occurred in 81. Minor complications were found in 15, and revision was performed in 4 . Sensitivity to touch was normal in all. Conclusion: Skin extension by multiple incisions is very easy to learn and can be performed without special instruments. The survival rate of the extended skin is high. Sensitivity to touch and mobility of the skin can be preserved, which results in a very durable nearly normal skin.
\end{abstract}

Following trauma, loss of skin and soft tissues, as well as extensive swelling, may hinder tension-free suture of the skin. Therefore, alloplastic materials such as Epigard ${ }^{\mathrm{TM}}$ or negative pressure vacuum seal were used for temporary wound coverage to allow swelling decrease. After an interval, these wound dressings were removed for secondary wound closure. Several surgical techniques like local rotational, expanding or transposition flaps, split- or full thickness skin grafts, and free flaps are described in the literature (1). Mostly split-thickness

This article is freely accessible online.

Correspondence to: Prof. Dr. med. Ralph Gaulke, Section Upper Extremity, Foot and Rheuma Surgery, Trauma Department, Medical School Hanover (MHH), Carl-Neuberg-Str. 1, D-30625 Hannover, Germany. Tel: +49 5115312015, e-mail: gaulke.ralph@mhhannover.de

Key Words: Soft tissue defect, wound closure, skin extension by multiple incisions, SEMI, local flap. skin graft is used, if the wound bed is vital. In cases with uncovered implants, bones or tendons, local or free flaps are indicated. Surgeons experience with the operative technique and the anatomy of flap perfusion is the key to success in these demanding procedures. Since 2007 the author uses the simple technique of skin extension by multiple incisions (SEMI) whenever possible. SEMI enables the contractile skin to be expanded by cutting the collagen fibers. After disconnection of the collagen fibers, the skin and the more flexible subcutaneous fat can be stretched easily to achieve a tension-free skin suture (Figure 1). While the subcutaneous layer is intact, bone, tendons and implants are safely covered to avoid deep wound infections. The multiple cuts allow the lymph to drain reducing swelling and improving tissue perfusion postoperatively. This study aimed to evaluate the preliminary clinical results of SEMI regarding wound healing, sensitivity and mobility.

\section{Patients and Methods}

This retrospective clinical study was performed with the approval of the local Ethics and Research Committee. All consecutive patients treated with SEMI by the author were included in the study as long as complete data of 100 SEMI were collected. Patients who received soft tissue coverage by combined techniques such as local rotational flaps with additional SEMI, with preoperative numbness of the skin around the operation field and those with incomplete data were excluded. The dimensions of the wound were documented intra-operatively before wound closure. The area of incisions was documented within five days postoperatively (Figure 2). Sensitivity to touch was tested in contrast to the opposite side after wound healing. Adhesions of the skin to fasciae, tendons and bones were evaluated clinically after complete healing of the skin at 12 weeks postoperatively.

Operative technique SEMI. The effect of the skin extension depends on the thickness and flexibility of the subcutaneous layer, the thickness and elasticity of the skin and the dimensions of the area were the incisions were performed. At the palm and the sole for example, subcutaneous flexibility is strongly limited by septation. In contrast to the palm and the sole, the subcutaneous fat layer at the forearm is very flexible and allows to cover much larger defects. That is why the surgeon should start to use this procedure at areas of flexible subcutaneous fat layer until he is firm with the limitations of this technique. 


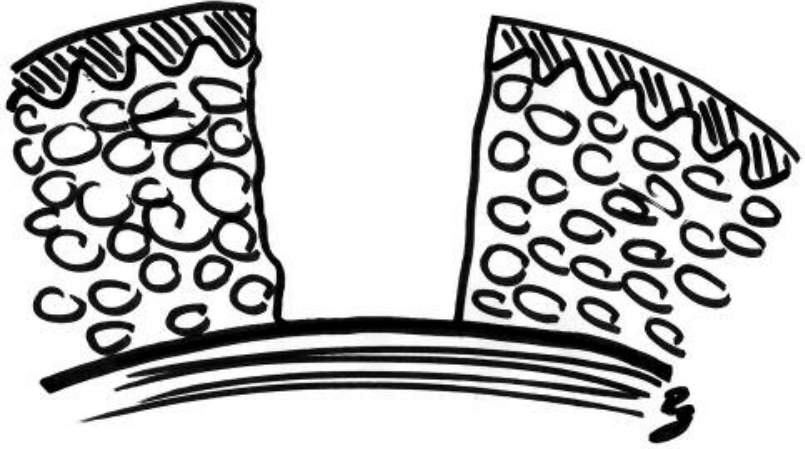

Figure 1. Effect of skin extension by multiple incisions (SEMI). (a) Soft tissue defect spread by the tension of the collagen fibers of the skin. (b) The elastic fibres of the skin were cut by multiple incisions. Wound closure is possible with mild tension on the wound margins.

The wound closure starts with the suture of the skin under tension. The tension will lead to strictures right-angled to the suture (Figure 3). Skin incisions with a length of three to five millimeters were performed perpendicular to the stricture, parallel to the wound margin. The skin incision spontaneously spreads and some venous blood my flow out. It is important to cut the skin as long as the incisions spread spontaneously. As soon as the soft tissue tension is reduced to normal, the SEMI is finished. The tension of the soft tissues must be normal at the end of the procedure (Figure 3 ). If the pressure is still high, a partial opening of the suture is essential to avoid skin necrosis or compartment syndrome. SEMI can be combined with rotation or extension flaps to enlarge the dimensions of these flaps as long as the tension is normal. Even in free flaps, SEMI helps to enlarge the flap and to drain the lymph.

The wound should be covered by fatty gauze, compresses and cotton wool ought to be used to absorb the blood and external pressure on the tissues has to be avoided. The wound dressing has to be changed on the first day postoperatively because the drying blood leads to stiffness of the dressing which may compromise the blood supply by squeezing the wound. From the first day, the wound dressing consists of compresses, cotton wool and elastic bandage. Further usage of fatty gaze may lead to maceration of the skin. For these reasons, daily changes of the dressing must be performed until the wound is dry. Sutures should not be removed too early to avoid wound dehiscence.

Statistics. The $t$-test and Wilcoxon rank-test were used to assess whether the width of the defect and SEMI are different and whether the width of SEMI-area influences the complication rate respectively.

\section{Results}

Enrolment and patient details. The data of 100 out of 129 consecutive SEMIs in 94 patients (59 women/35 men) were included. The mean age at surgery was 46.5 years (median=47.5; range $=15-82 ; 95 \% \mathrm{CI}=43.44-49.52$ ). Fifty-five defects were located on the right side and 45 on the left side. SEMI was mostly located at the forearm, followed by foot and ankle, lower leg, elbow and upper arm, thigh and knee. a

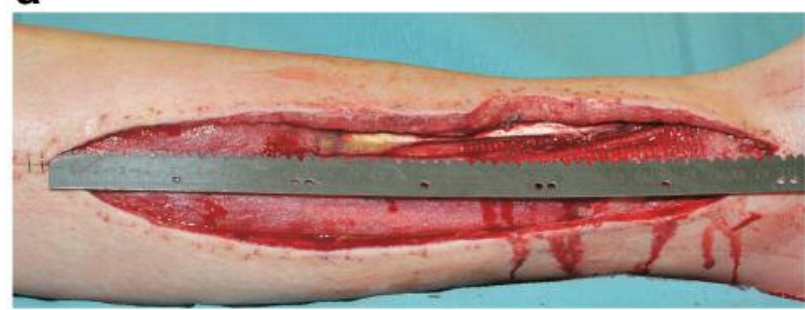

b

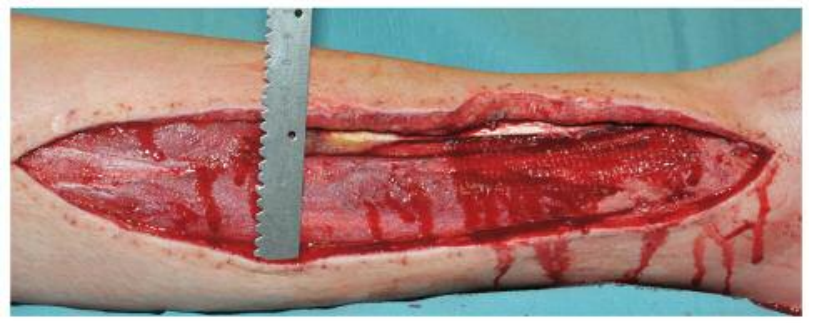

C

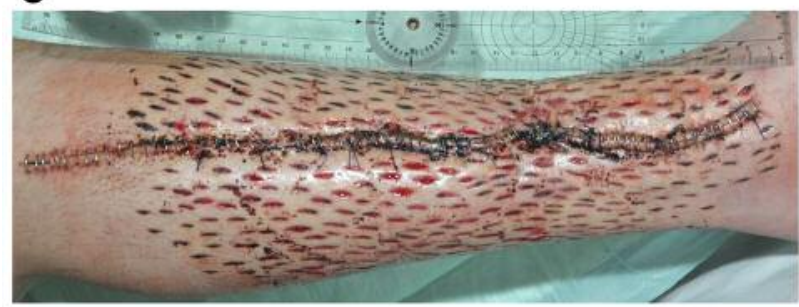

d

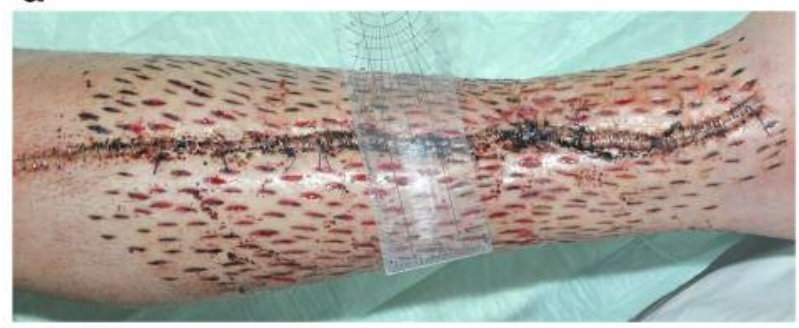

Figure 2. Wound closure at the lower leg. $(a, b)$ Soft tissue defect after compartment release at the lower leg. (a) Length: $30 \mathrm{~cm}$, (b) width: 6 $\mathrm{cm} .(\mathrm{c}, d)$ SEMI at the fifth day postoperatively. (c) length $30 \mathrm{~cm}$, (d) width: $12 \mathrm{~cm}$.

Fourteen SEMI extended over two regions of the extremity, were mostly located at the forearm and dorsum of the hand. One SEMI spread out from the thigh and lower leg to the dorsum of the foot. In 6 patients ( 1 woman $/ 5$ men) defects on two sites were closed by SEMI (Figure 4).

From 129 consecutive SEMI in 123 patients, 24 were performed together with other local flaps and another three were excluded due to the numbness of the skin preoperatively. In another two patients, the data were incomplete (Figure 4).

Defect surface. The mean surface of all 100 defects was $22.6 \mathrm{~cm}^{2}$ with a wide range from $4 \mathrm{~cm}^{2}$ to $184.5 \mathrm{~cm}^{2}$ 
a

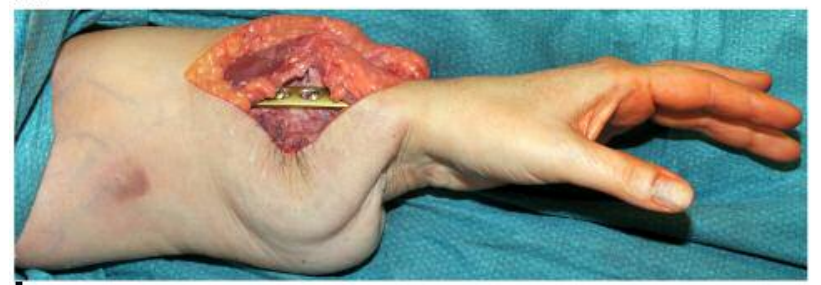

b

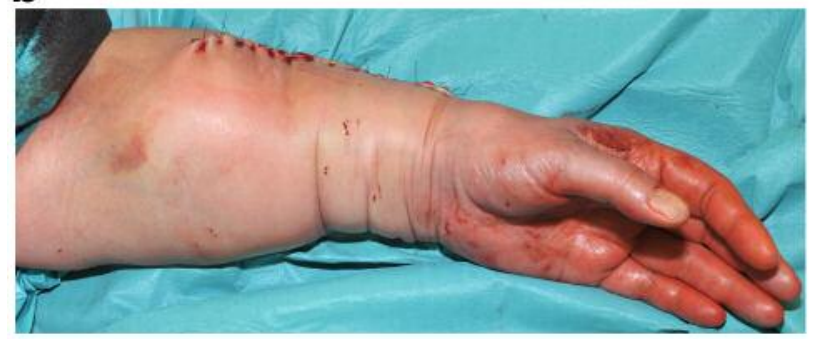

C

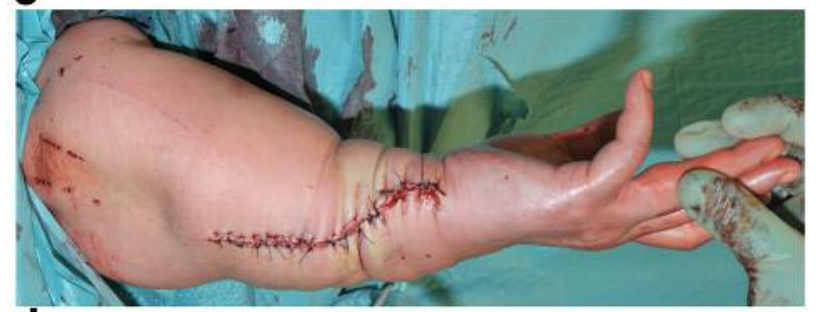

d

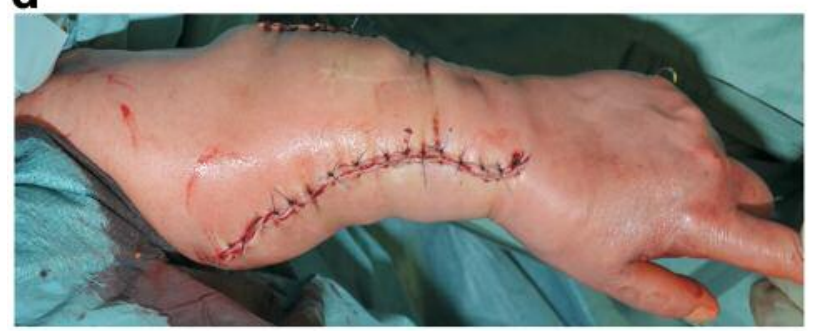

e

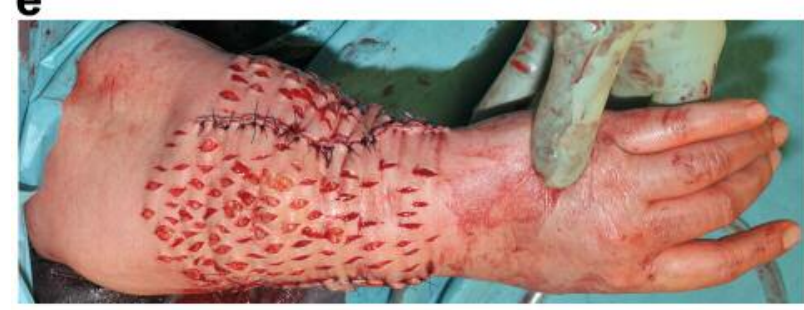

f

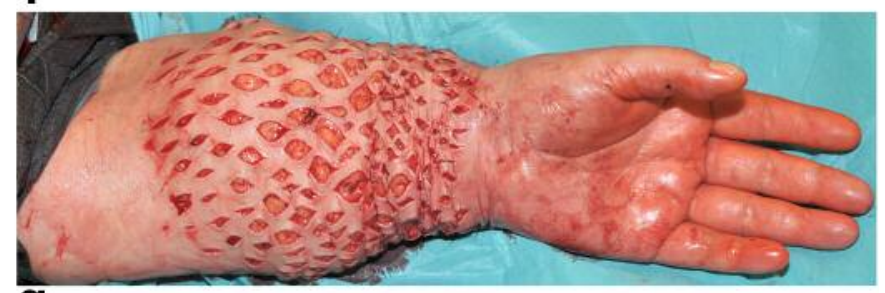

g

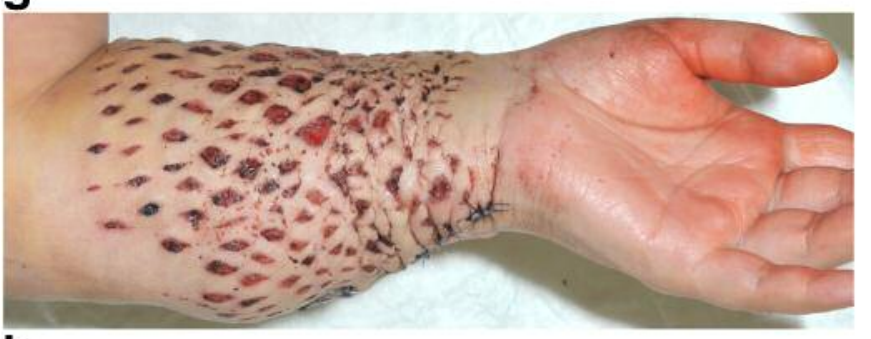

h

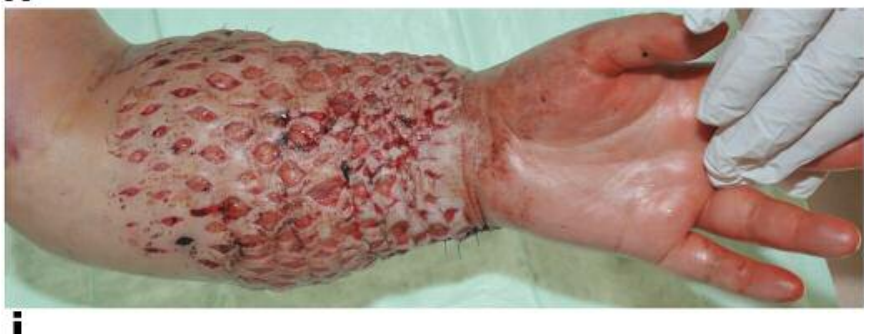

i
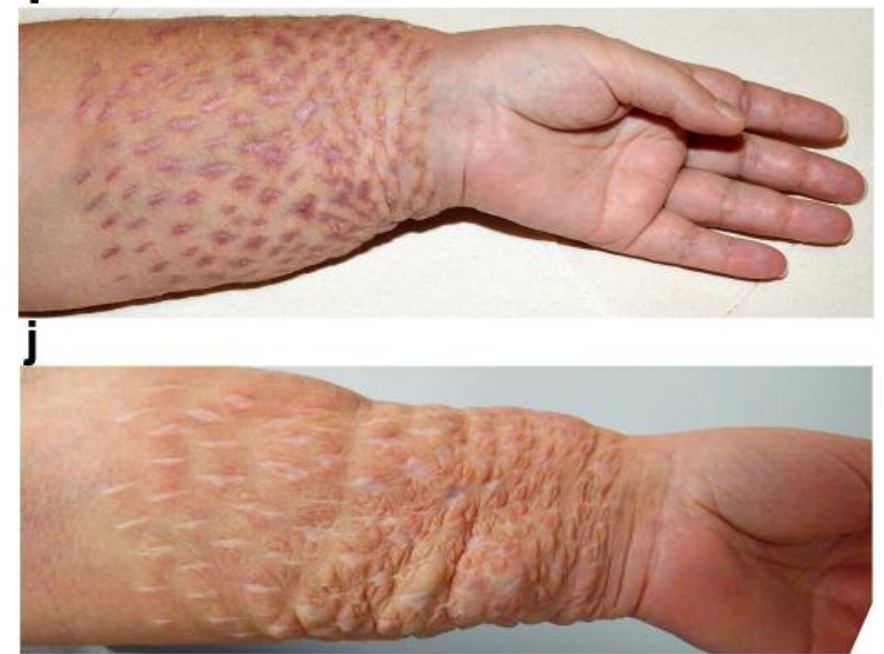

Figure 3. Wound closure after shortening of the forearm. (a) Surplus of soft tissues after shortening of the forearm for recurrent non-union of the ulna and radial shaft. $(b-d)$ Skin stricture led to strangulation of the soft tissues at the distal forearm after wound closure. (e,f) SEMI reduces the strangulation and allows soft tissue swelling. (g) First day after surgery, swelling is reduced. (h) Nine days after surgery: the cuts with the lowest skin tension at the margins of SEMI start shrinking which leads to spontaneous closure of the incisions. (i) Eight weeks after surgery the scars are still active. (j) 20 months postoperatively scarring is completed.

(median $=15 ; 95 \% \mathrm{CI}=19.56-25.64)$. The mean longitudinal dimension was $10.1 \mathrm{~cm}$ (median=7.8; range $=4-41 \mathrm{~cm}$; $95 \% \mathrm{CI}=10.0-10.2)$. The mean width of the defect was 3.9 $\mathrm{cm}($ median $=4.0$; range $=2$ to $7 \mathrm{~cm} ; 95 \% \mathrm{CI}=3.88-3.92)$.
SEMI surface. The mean surface of the skin area treated with SEMI was $65.2 \mathrm{~cm}^{2}$ (median $=35$; range $=7-1050 \mathrm{~cm}^{2}$; 95\% CI=42.28-63.44). The mean area of incisions was more than twice as large as the defect. Because of the wide range 


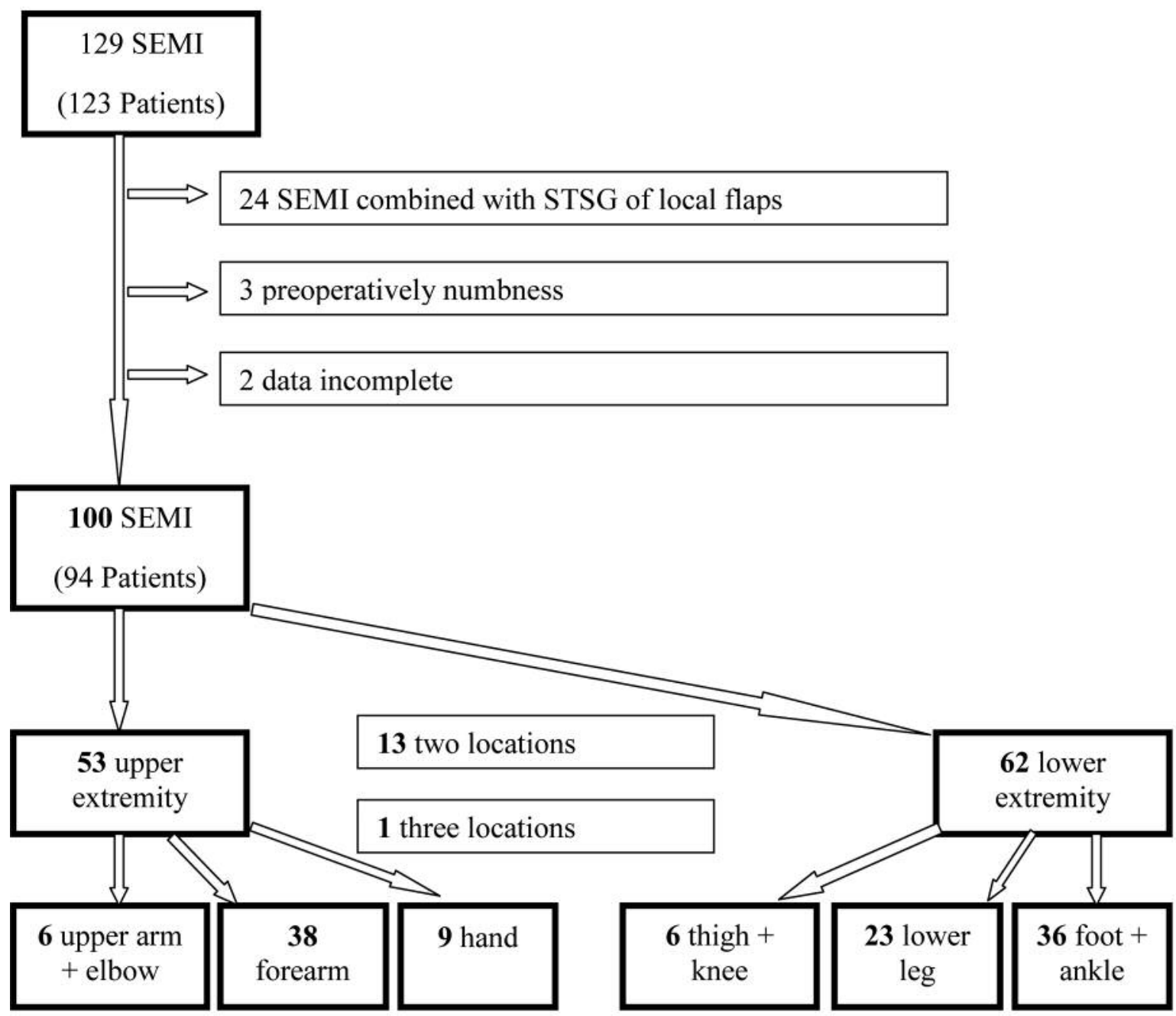

Figure 4. Incidence and localization of SEMI at the extremities. Thirteen lesions expanded over two locations, one over three.

this difference was not statistically significant $(p<0.15)$. The mean longitudinal dimension was $10.4 \mathrm{~cm}$ (median=8.3; range $=2-70 \mathrm{~cm} ; 95 \% \mathrm{CI}=9.54-11.36$ ). The longitudinal dimension of SEMI and soft tissue defect did not differ significantly $(p<0.9)$. The mean width of SEMI, rectangular to the suture of the wound, was $9.6 \mathrm{~cm}$ (median $=8.0$; range $=3-30 \mathrm{~cm} ; 95 \% \mathrm{CI}=8.79-10.47$ ), which is about 2.5 -fold larger than the soft tissue defect. The skin area with the incisions is significantly wider than the soft tissue defect $(p<0.001)$.

Complications. In 81 cases wound healing was regular. Superficial necrosis of the wound margin was found in 15 wounds of 14 patients, all healed under conservative treatment (Figure 5). Another four wounds needed revision for deep wound infection. All but one healed after local revision and primary wound closure. Debridement and split skin graft were performed in one patient. The mean surface of SEMI with necrosis at the wound margins was $117.5 \mathrm{~cm}^{2}$ (median=58 $\mathrm{cm}^{2}$; range $=17.5-1,050 \mathrm{~cm}^{2}$; 95\%CI=93.29141.71), while the mean surface of SEMI with regular wound healing was smaller (mean: $52.9 \mathrm{~cm}^{2}$; median: 30 ; range $\left.=7-307.5 \mathrm{~cm}^{2} ; 95 \% \mathrm{CI}=51.21-54.59\right)$.

Sensitivity to touch and adhesions. A loss of sensitivity to touch compared to the surrounding skin and the skin of the collateral side was not found. Adhesions of the skin to deep soft tissue layers such as muscle, tendon or bone were not found. The mobility of the skin was comparable to the surrounding and the contralateral skin in all patients. 
a

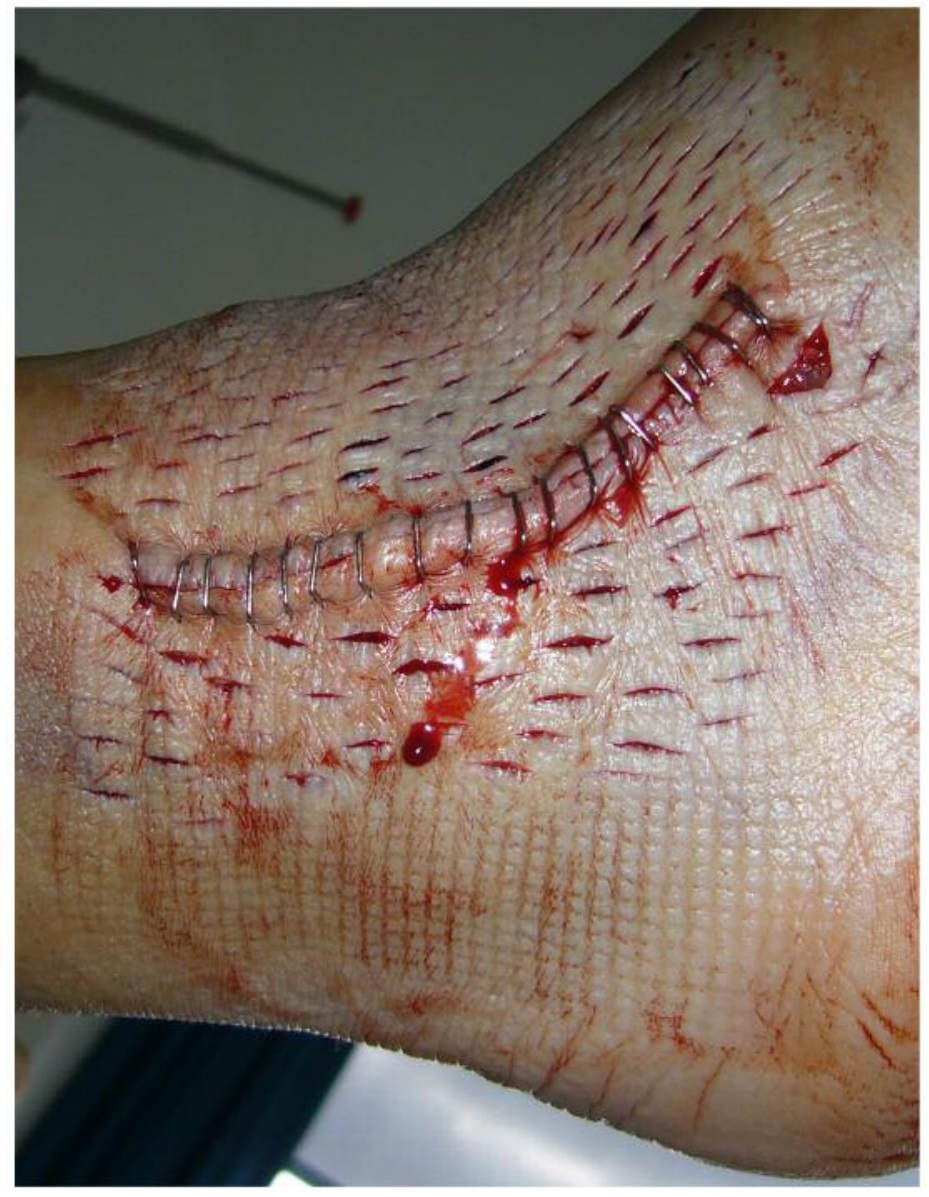

b

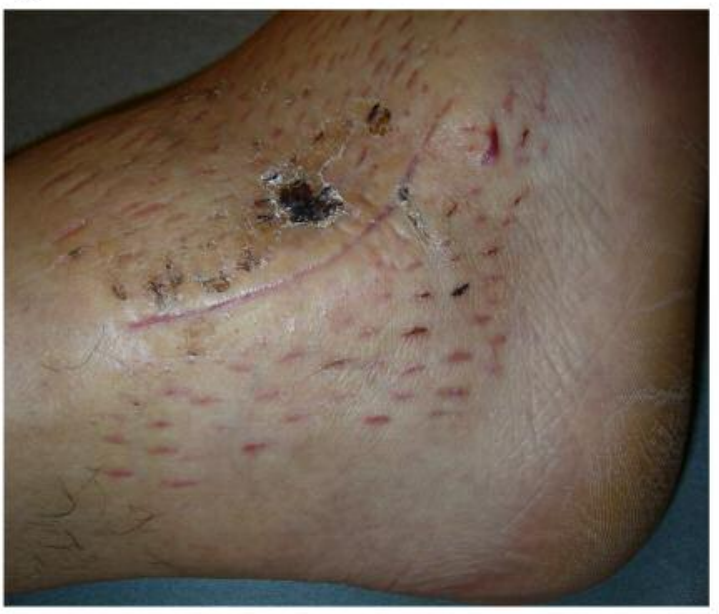

C

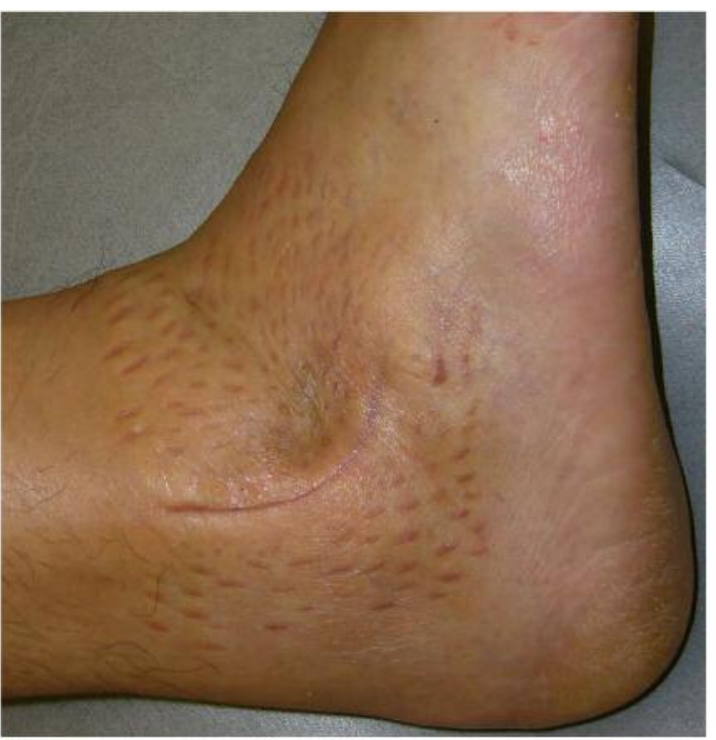

Figure 5. Conservative treatment of skin necrosis. (a) First day after SEMI and osteosynthesis of the lateral malleolus. (b) Superficial skin necrosis after 1 month, (c) secondary healing of the skin at 2 months.

\section{Discussion}

The current investigator sought to evaluate the preliminary clinical outcome of skin extension by multiple incisions (SEMI) (2). Complications such as disturbed wound healing, need for secondary surgery, and numbness of the skin were documented.

To the knowledge of the author, the technique of SEMI has not yet been described for wound closure in the literature.

Raveendran et al. described multiple stab incisions to evacuate contrast extravasation of the hand and forearm in a case report (3). In contrast to the technique described in our study, the cuts they described in their case report were much deeper, reaching subcutaneous extravasation to eliminate toxic contrast medium. The surgical technique and the indication differ from superficial skin extension for wound closure.

The most common technique for the coverage of soft tissue defects, that can not be directly sutured, is splitthickness skin graft. This technique left a donor site scar that is cosmetically relevant through the loss of pigmentation. Kanapathy and Mosahebi compared split- to full-thickness skin grafts in five patients each and found that the skin suture following full-thickness skin graft (FTSG) is more 
aesthetic than the donor site defect after split-thickness skin graft (STSG). However, they did not examine the grafts (4). SEMI leaves multiple small scars without adhesions to the underlying tissues like fasciae, tendons or bone and no tendency to contract. There was no additional donor site defect with this technique.

Adams and Ramsey reviewed the complaints following FTSG and STSG (5). They also found that wound healing at the donor site is prolonged after STSG. The cosmetic result after FTSG is superior to STSG at donor sites of radial forearm flaps, both grafts develop numbness and may contract (6). Ulceration may occur through the lack of sensitivity, especially at the lower leg and the foot.

To restore skin sensitivity after FTSG and STSG, skin expanders (silicon bags) were implanted epifascially underneath the subcutaneous layer and were successively percutaneously filled with sodium solution. As the reservoir was filled, the graft was removed and the defect was covered with the expanded skin. Complications like infection of the expander and wound dehiscence are common. Osmotically active hydrogel expanders have been used alternatively to silicon bags. The use of expanders is strongly limited by the remaining tension of the elastic fibers of the skin $(7,8)$.

Local or neurovascular pedicled flaps should be used whenever possible to preserve sensitivity to touch. The subcutaneous fat enables the skin to glide over the deeper tissues such as fasciae, tendons and bones. Partial and total flap necrosis may appear up to $20 \%$ at the extremities depending on local blood supply (9). The surgical technique in local flaps is more difficult than in free skin grafts but easier than in vascular anastomosed free flaps. Free flap surgery is demanding and special instruments and optical devices, like a microscope, are needed. Nevertheless, complications following free flaps are common with revision rates about 15-20\% (10, 11). Fasciocutaneous flaps have a higher revision rates but even salvage rates compared to musculocutaneous flaps (12). Donor site defects even in muscle flaps may also cause complications like seroma, hematoma, deep infection and a loss of strength.

These high revision rates show the worth of SEMI, which helps to spare local and free flaps.

Limitations of this study are the retrospective design, the variety of sites and dimensions of SEMI, and the fact that the author demonstrates the results of his therapeutic strategy. Therefore, prospective studies on SEMI for each region of the body compared to other techniques in plastic surgery such as free skin grafts, other local and free flaps may help to evaluate the limitations of this promising and easy to learn procedure.

\section{Conclusion}

SEMI is an easy-to-learn surgical technique for wound closure of soft tissue defects with no need for special instruments or optical devices. A rational usage of this technique may help spare demanding procedures with donor site defects and higher complication rates.

\section{References}

1 Kinzl L, Suger G and Stober R: Covering soft tissue defects. Unfallchirurg 99: 714-726, 1996.

2 Gaulke R: Diaphyseal fractures of the forearm. In: Skeletal trauma 5th Ed. Browne DB, Jupiter JB, Krettek C, Anderson PA (eds.). Philadelphia, Elsevier, pp. 1313-1346, 2015.

3 Raveendran S, Rajendra Benny K, Monica SA, Pallapati SR, Keshava SN and Thomas BP: Multiple stab incisions and evacuation technique for contrast extravasation of the hand and forearm. J Hand Surg Am, 2018. doi 10.1016/j.jhsa.2018.08.009 [Epub ahead of print]

4 Kanapathy $\mathrm{M}$ and Mosahebi A: Comparative study on the dodnorsite aesthetic outcome between epidermal graft and split thickness skin graft. Int Wound J, 2018. doi 10.1111/iwj.13039 [Epub ahead of print]

5 Adams DC and RamseyML: Grafts in dermatologic surgery: review and update on full- and split-thickness skin grafts, free cartilarginal grafts, and composite grafts. Dermatol Surg 31: 1055-1067, 2005.

6 Davis WJ 3rd, Wu C, Sieber D and Vandevender DK: A comparison of full and split thickness skin grafts in radial forearm donor sites. J Hand Microsurg 3: 18-24, 2011.

7 Pamplona DC, Weber HI and Leta FR: Optimization of the use of skin expanders. Skin Res Technol 20: 463-472, 2014.

8 Bergé SJ, Wiese KG, von Lindern JJ, Niederhagen B, Appel T and Reich RH: Tissue expansion using osmotically active hydrogel systems for direct closure of the donor defect of the radial forearm flap. Plast Reconstr Surg 108: 1-5, 2001.

9 Qian Y, Li G, Zang H, Cao S, Liu Y, Yang K, Mu L: A systematic review and meta-analysis of free-style flaps: risk analysis of complications. Plast Reconstr Surg Open 6: e1651, 2018.

10 Bigdeli AK, Gazyakan E, Schmidt VJ, Bauer C, Germann G, Radu CA, Kneser U and Hirche C: Long-term outcome after successful lower extremity free flap salvage. J Reconstr Microsurg, 2018. doi 10.1055/s-038-1675146 [Epub ahead of print]

11 Stranix JT, Jacoby A, Lee ZH, Anzai L, Saadeh PB, Thanik V and Levine JP: Skin paddles improve muscle flap salvage rates after microvascular compromise in lower extremity reconstruction. Ann Plast Surg 81: 68-70, 2018.

12 Stranix JT, Lee ZH, Jacoby A, Anzai L, Mirrer J, Avraham T, Thanik V, Levine JP and Saadeh PB: Forty years of lower extremity take-backs: flap type influences salvage outcomes. Plast Reconstr Surg 141: 1282-1287, 2018. 